\title{
Riding the Cell Jamming Boundary: Geometry, Topology, and Phase of Human Corneal Endothelium
}

\author{
Nigel H. Brookes
}

New Zealand National Eye Bank and Department of Ophthalmology, University of Auckland, New Zealand.

\author{
n.brookes@auckland.ac.nz
}

\begin{abstract}
It is important to assess the viability of eye-banked corneas prior to transplantation due to inherent senescence and known loss of endothelial cells during surgical manipulation. Corneal endothelial cells have a complex basal and paracellular shape making them challenging to accurately measure, particularly in oedematous ex vivo tissue. This study used calibrated centroidal Voronoi Diagrams to segment cells in images of these human corneas, in order to characterize endothelial geometry, topology, and phase.

Hexagonal cells dominated the endothelia, with most comprised of five different pleomorphs exhibiting selfsimilar topological coarsening through most of the endothelial cell density range. There was a linear relationship between cell size and shape, though cells with greater than six sides were present in larger proportions than cells with less. Hexagonal cell regularity was stable and largely independent of density.

Cell and tissue phase was also examined, using the cell shape index relative to the recently discovered 'cell jamming' phase transition boundary. Images showed fluid endothelia with a range of shape indices spanning the boundary, independent of density but dependent on hexagonal regularity. The cells showed a bimodal distribution centred at the boundary, with the largest proportion of cells on the fluid side. A shoulder at the boundary suggested phase switching via shape transformation across the energy barrier, with cells either side having distinctly different size and shape characteristics. Regular hexagonal cells were closest to the boundary.

This study showed the corneal endothelium acts as a glassy viscous foam characterized by well-established physical laws. Endothelial cell death transiently and locally increases cell fluidity, which is subsequently arrested by jamming of the pleomorphically diverse cell collective, via rearrangement and shape change of a small proportion of cells, which become locked in place by their neighbours and maintain endothelial function with little energy expenditure.
\end{abstract}

\section{Keywords}

Cornea, Human, Endothelium, Geometry, Topology, Cell Jamming, Voronoi Diagram, Organ Culture
Abbreviations
CJB: Cell Jamming Boundary
CV: Coefficient of Variation 
CVT: Centroidal Voronoi Tessellation

ECD: Endothelial Cell Density

SD: Standard Deviation

SEM: Standard Error of the Mean

SI: Shape Index

VD: Voronoi Diagram

\section{Introduction}

\subsection{Microscopy and Image Analysis}

Recent immunohistochemical studies have confirmed that corneal endothelial cells have a complex shape, comprised of foam-like apical pole coupled to a complex interdigitating stellate basal pole that is tightly anchored to Descemet's membrane [Harrison et al., 2016; He et al., 2016]. Traditional examination of only the apical view in vivo with a specular or confocal microscope can only provide limited information about the endothelium, or the cornea as a whole.

The ex vivo corneas examined in this study were organ-culture stored and assessed using hypotonic sucrose to swell and make their intercellular borders visible [He et al., 2016; Kirk and Hassard, 1969; Pels, 1997; Sperling, 1980]. While this makes the complex basal morphology more evident, it also makes morphometric analysis more difficult using common edge-based segmentation techniques, so every segmentation boundary is an approximation. This has led to the alternative approach used in this study, where point clouds of cell centroids were used to generate calibrated Voronoi Diagrams (VD), which define the region occupied by each cell and their borders [Brookes, 2017a]. Endothelial polymegathism and pleomorphism was measured using the size and shape of these regions, facilitating the large-scale examination of corneal endothelial geometry, topology and phase [Brookes, 2017b].

\subsection{Cell Density and Shape}

The viability of an eye-banked cornea for transplantation is usually quantified by microscopically determining endothelial cell density (ECD) [Wilson and Bourne, 1989], which declines constantly throughout life [Wilson and Roper-Hall, 1982]. While the density of the endothelial molecular pumps has been shown to remain remarkably constant in order to maintain normal corneal thickness and transparency [Geroski et al., 1985], corneal decompensation and failure can result when the ECD drops to very low levels [Mishima, 1982, Nishimura, 1999]. This is a problem as much corneal tissue stored for transplant is from older donors [Cunningham et al., 2012], and transplantation surgery inevitably causes cell loss, particularly popular lamellar procedures such as Descemet's Stripping Endothelial Keratoplasty [Price and Price, 2008].

This steady decrease in ECD throughout life is due to the inherent senescence of the endothelial cells, which in vivo are arrested in the G1-phase of the cell cycle, and dead cells are not replaced. Three mechanisms have previously been identified that contribute to this senescence: cell-cell contact-dependent inhibition correlated to the time endothelial cell division ceases and mature cell contacts form, lack of effective growth factor stimulation, and TGF- $\beta 2$ suppression of the S-phase of the cell cycle [Joyce, 2005; Joyce, 2012].

Beyond ECD, the polymegathism and pleomorphism of the endothelial cells was recognized in early studies to also affect corneal viability. For example, corneal endothelia from diabetic patients showed abnormalities 
in cell size, shape, and arrangement, suggesting these may be early indicators of endothelial distress [Rao, 1985; Shultz et al., 1984], and the decline in ECD and the proportion of hexagonal cells after corneal transplantation appeared to affect the development of late endothelial failure [Bourne, 2001].

During early gestation, there is a rapid increase in the density of endothelial cells to about $16,000 \mathrm{cells} / \mathrm{mm}^{2}$, but as gestation proceeds the corneal diameter increases and this density steadily decreases. At birth, the corneal endothelium was long assumed to be a uniform monolayer comprised mostly of hexagonal cells, but more detailed analysis has shown that while it has a cell density of $4000-6000$ cells $/ \mathrm{mm}^{2}$ [Elbaz et al., 2017; Ko et al., 2001] there is also a high level of pleomorphism [Doughty, 1989; Müller et al., 2000]. Cell density values in children are significantly correlated to corneal diameter [Elbaz et al., 2017; Müller and Doughty, 2002; Murphy et al., 1984]. This trend continues through life, with a steady decrease in hexagonal cells, and a corresponding increase in pentagonal and heptagonal cells [Yee et al., 1985]. A study of corneal endothelia over 100 years of age found a mean cell density of $923 \mathrm{cells} / \mathrm{mm}^{2}$ comprised of about $55 \%$ hexagonal cells [Ruusuvaara et al., 2015]. There are also significant differences in cell density and percentage of hexagonal cells in the central, paracentral, and peripheral endothelium [Amann et al., 2003; Schimmelpfennig, 1984].

As a tessellated matrix of perfectly regular hexagons can only tile a flat plane, the curved posterior corneal surface must have a more complex pleomorphic composition from the outset. Tessellation of negative curvatures tends to increase the proportion of cells with more than six sides, compared to cells with less [Weaire and Rivier, 1984].

A variety of statistics have been used to characterize the composition and disorder of the corneal endothelium. The most common are the percentage of hexagonal cells and the Coefficient of Variation (CV) of the cell size, however both these are problematic. Large CV values may be due to disproportionately smaller or larger cells of unknown polygonality [Doughty, 1990; Doughty, 2013], and the percentage of hexagonal cells provides no information on their symmetry or regularity [Doughty, 1992]. The calculation of $\mathrm{CV}$ of the cell area is programmed into the analysis software in many commercial specular microscopes and continues to appear in the cornea literature.

Alternative measures also appearing in the literature include the figure coefficient (or shape factor), shape deviation index, skewness index, hexagon deviation index [Alanko, 1983; Doughty, 1989; Doughty, 1992], area-side plots [Dilts and Doughty, 1996; Doughty and Dilts, 1994], and angular regularity [da Fontoura Costa, 2006], but none of these have been widely used. The area-side plots are useful as they show a linear relationship between the cell area and the number of cell sides, as long as the sample size is large enough [Doughty, 1998a]. This is a demonstration of the Lewis law, where the average area of an $n$-sided cell in a two-dimensional lattice increases linearly with $n$, so small cells tends to have fewer sides [Lewis, 1926]. This law has general application in many other fields beyond corneal endothelial architecture [Chiu, 1995].

In addition to the Lewis law a number of other factors also determine the relationship between cell polygonality and size/density in a two-dimensional tessellated lattice. In general it is also defined by Euler's formula in two dimensions ( $n_{\text {cells }}-n_{\text {edges }}+n_{\text {vertices }}=$ Euler constant; in a tissue with a large number of cells the average number of neighbours of a cell will be six) [Cantat et al., 2013], the Aboav-Weaire law (cells with a higher number of sides tend to have few-sided neighbours and vice versa), and the von Neumann-Mullins relation (a cell with more than six sides grows and a cell with less than six sides shrinks, with equilibrium being obtained by hexagonal cells). Much of this understanding has been developed through the study of analogous non-biological systems, such as foams and mathematical modelling [Cantat et al., 2013; Chiu, 1995; MacPherson and Srolovitz, 2007; Sánchez-Gutiérrez et al, 2016; Weaire and Rivier, 1984; Wörner et al., 2011]. 


\subsection{Tissue Organization}

Tissue homeostasis occurs through the interplay between a robust and stable architecture that can resist stress, with one exhibiting sufficient plasticity to allow remodelling to maintain tissue function [Lecuit and Lenne, 2007]. The basic laws of organization of groups of cells in a tissue were first identified by Plateau in 1873 , and the apical surface of the endothelial cells will tend toward equilibrium of faces (smooth surfaces with a constant mean curvature determined by the Young-Laplace law) and edges (surfaces always meet in threes at $120^{\circ}$ angles) [Cantat et al., 2013]. At equilibrium, the tissue is at an energy minimum and cells tend to aggregate in clusters, where the surface area in contact with the surrounding environment is minimized [Lecuit and Lenne, 2007]. In the minimal surface model this is the point where the total length of all cell edges is at a minimum and is determined solely by the cell geometry [Weaire and Rivier, 1984], though Plateau's laws have been shown to break down in foams as the liquid fraction increases [Drencken and Hutzler, 2015]. In vivo, the cell geometry is further determined by both intracellular and extracellular mechanics, including cell-to-cell and cell-to-substrate adhesion, cytoskeleton, surface tension and osmotic swelling, which all act as energy barriers preventing the system from relaxing to the global minimum of energy.

Cellular dynamics have been modelled in many tissues including corneal endothelium [Honda et al., 1982], vascular endothelium [Vitorino et al., 2011], and keratinocyte monolayers [Bock, 2010]. Cells autonomously migrate within the monolayer and turn in response to mechanical cues resulting from adhesive, drag, repulsive, and directed steering interactions with neighbouring cells. Intracellularly, actin-based protrusions such as filopodia and lamellipodia have long been accepted as key constituents in these processes, together with cellular blebbing in areas of weak membrane-cytoskeleton attachment [Charras et al., 2008; Norman et al., 2010; Norman et al., 2011; Reinhart-King et al., 2005]. There is a tendency for the local migration at the mesoscale to follow the local orientation of maximum principal stress, called plithotaxis [Pegorano et al., 2016]. This mechanism of collective cell guidance is an emergent property that requires cooperativity of mechanical stresses across many cell-to-cell junctions [Trepat and Fredburg, 2011; Sadati 2013]. In contrast, individual cell death as in the corneal endothelium causes neighbouring cells to exert a traction stress aligned toward the void, via a phenomenon called kenotaxis [Kim et al., 2013; Park et al., 2016]. This is also seen on larger scales during wound healing [Honda et al., 1982].

Changes in a range of tissues that involve remodelling of cell-cell contacts have been shown to be accompanied by an increase in (Shannon) entropy in the form of both geometric and topological disorder, by progressively shifting a mosaic from a hexagonal arrangement (the VD generated from a perfectly triangular lattice of centres) to a perturbed one with increased polymegathism and pleomorphism [Lecuit and Lenne, 2007, Mason et al., 2012, Weaire and Rivier, 1984]. While this final organization may be irregular it is not random [Doughty, 1998b].

As the cornea ages, every endothelial cell death transiently increases tissue entropy and fluidity, forcing cells to rearrange in order to maintain endothelial function. In the vertex model, a system that has been used extensively to model monolayers, these cell neighbour exchanges are known as $\mathrm{T} 1$ processes or transitions that occur via successive boundary shortening procedures. Cell death occurs as a series of T1 processes followed by a T2 process removing the last part of the cell [Honda and Nagai, 2015, Cantat et al., 2013; Weaire and Rivier, 1984]. These initially kenotactic rearrangements occur in response to a non-equilibrium state, causing tissue relaxation via von Neumann-Mullins coarsening, at a rate controlled by the amount of energy required to execute them, and the degree of collective cell fluidity [Bi et al., 2014]. These processes maintain Plateau's laws and Euler's formula, and also leave the topological charge of the tissue averaging zero [Cantat et al., 2013]. 
Much of the physical tissue properties are determined by the geometry and topology of the cells comprising it. A cell's ability to move within the tissue is topologically constrained by its neighbours, and the number and arrangement of these neighbours in turn define its geometry. Recently a 'cell jamming' phase transition boundary (solid-fluid; CJB) that characterizes this glassy collective cell dynamic has been discovered in the vertex model, and confirmed in a Voronoi model [Bi et al., 2016]. It can be measured with the cell shape index (SI), a dimensionless parameter that is the ratio of the cell perimeter to the square root of the cell area, that effectively encodes the physical properties of each cell such as cell-cell adhesion and cortical tension [Bi et al., 2015]. Sl's higher than 3.81 define a fluid cell collective, and below, a solid (jammed) one. For example, in cancer biology this parameter can differentiate between a benign (solid) and a metastasizing (fluid) tumour, essentially encoding the epithelial-mesenchymal transition [Haeger et al., 2014]. Similarly, cultures of asthmatic cells from the airway epithelium retain their fluidity much longer than non-asthmatic cells [Park et al., 2015, Park et al., 2016]. The phase dynamics of the human corneal endothelium are currently unknown.

\subsection{Summary}

This study extends the dataset generated previously [Brookes, 2017a] with the additional calculation of SI for each cell, analysed in relation to cell density, size, and shape, in order to characterize endothelial tissue geometry, topology, and phase in ex vivo human organ-culture-stored corneas [Brookes, 2017b].

\section{Methods}

The dataset used in this study was further developed from one described previously [Brookes, 2017a]. Measurements of 354,998 cells were made from 2000 routine endothelial assessment images, taken after organ-culture storage but prior to release for transplantation, from a sequential series of 678 corneas from 351 donors, that were collected with consent by the New Zealand National Eye Bank over several years [Brookes, 2017b]. Images were analysed using custom MacOS software, with manually marked cell centroids within a fixed frame or overlapping right or bottom borders. Centroids of cells outside the frame were automatically detected, and the full point cloud used to generate a calibrated VD for the entire image field. These Voronoi 'cells' mapped to the observed endothelial cell borders and were used to calculate cell area and shape. Detailed statistics examined in this current study were:

\section{Polymegathism}

- ECD of each image (cells $\left./ \mathrm{mm}^{2}\right)$

- Coefficient of variation (CV; standard deviation/mean) of the cell area in each image

- Cell area of all cells $\left(\mu \mathrm{m}^{2}\right)$

- Cell area of hexagonal cells $\left(\mu \mathrm{m}^{2}\right)$

Pleomorphism

- 4-9 sides mean percentage area in each image

- Mean number of pleomorphs (different polygonal shapes in the image)

- Mean number of cell sides in each image

- $\mathrm{CV}$ of the number of cell sides in each image 
- Number of sides of all cells

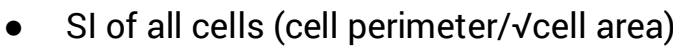

- Regularity of hexagonal cells (percentage difference in size ratio from a regular hexagon with short/long side diameter ratio of $\sqrt{ } 3 / 2=0.866$ )

Tabulated size and shape data for all images and cells was transferred via tab-delimited text files into Microsoft Excel for analysis. For each image, ECD data was ranked, grouped, and the mean and standard error of the mean calculated for each corresponding group of shape data (pleomorph percentage areas, hexagonal regularity, number of pleomorphs, number of sides, coefficient of variation of the cell area and number of sides, $\mathrm{SI}$ ). For each cell, cell area was ranked, grouped, and the mean, standard error of the mean (SEM), and standard deviation (SD) calculated for each corresponding group of shape data (number of sides, $\mathrm{SI}$ ). For each hexagonal cell, hexagonal cell area was ranked, grouped, and the mean, SEM, and SD calculated for each corresponding group of shape data (hexagonal regularity, $\mathrm{SI}$ ). The normality and skewness of the resulting data distributions were determined using SPSS [IBM Corp. Released 2011. IBM SPSS Statistics for Macintosh, Version 20.0. Armonk, NY: IBM Corp.], and finally all data plotted using Prism [Version 6 for Mac OS X, Graphpad Software, Inc., USA].

\section{Results}

\subsection{Images}

The image ECD data [7] was found to have a non-normal distribution (1-sample Kolomogorov-Smirnov test; $p$ $=0)$ skewed negatively (-0.695), as plotted in Graph 1 .

Image Mean \pm SEM shape data (pleomorph 4-9 percentage areas, number of pleomorphs, number of sides, $\mathrm{CV}$ of the number of sides and mean cell area), was plotted against the full range of ECD values in Graphs 25. Data from the single outlier image with mean density greater than 4500 cells $/ \mathrm{mm}^{2}$ was not plotted. Graphs 3-5 were plotted using a limited $y$-axis subset to illustrate more subtle trends.

\subsection{Cells}

The cell area and number of sides data from the both all 354,998 cells and the subset of 182,344 hexagonal cells was ordered and grouped according to the relationship: cell area $\left(\mu \mathrm{m}^{2}\right)=1,000,000 \div \mathrm{ECD}\left(\mathrm{cells} / \mathrm{mm}^{2}\right)$ [7]. The area data of both groups of cells had a non-normal distribution (1-sample Kolomogorov-Smirnov test; $p=0$ ) and positive skew (all cells: 2.241 ; hexagonal cells: 2.298 ). The cell area distributions were plotted in Graph 6 . The cell area grouping and ordering in this graph was arranged to directly compare with the ECD grouping in Graphs 1-5.

The relationship between cell area and number of sides was plotted in Graph 7 as a line graph using a limited $y$-axis for comparison to Doughty's data [Doughty 1998b], and a linear regression line calculated ( $y=$ $\left.41.24 x+105.7 ; r^{2}=0.997 ; p<0.0001\right)$.

The regularity of the subset of hexagonal cells relative to their cell area was plotted in Graph 8 , which has a limited $y$-axis subset to illustrate more subtle trends. Again, the cell area grouping and ordering in this graph was arranged to directly compare with the ECD grouping in Graphs 1-5. 


\subsection{Shape Index}

The image SI Mean \pm SD $=3.87 \pm 0.02$ (Range: $3.81-3.93$ ). The distribution of the mean image SI is plotted in Graph 9.

The relationship of the cell SI to mean image ECD and \% area of hexagonal cells was plotted in graphs 10-11. The dotted line at a SI of 3.81 in these graphs represents the CJB [64]. Shape indices above this line indicate collective cell fluidity. The SD was plotted here to show the spread of cell SI values in relation to the CJB. Graphs 10-11 were plotted using a limited y-axis subset to illustrate more subtle trends.

The SI distribution at the cell level was plotted in Graph 12. The cell SI Mean \pm SD $=3.87 \pm 0.09$ (Range: 3.65 $-4.72)$.

The cell populations under at the data points at the bimodal peaks either side of the CJB in Graph 1 were further analysed, and results summarized in Table 1 and Graphs 13-14. The cell area grouping and ordering in Graph 13 was also arranged to directly compare with the ECD groupings in other graphs.

\begin{tabular}{|r|l|l|}
\hline & Solid $(\mathrm{SI}=3.80)$ & Fluid $(\mathrm{SI}=3.86)$ \\
\hline Number of Cells: & 15005 & 16808 \\
\hline Cell Area (Mean \pm SEM): & $370.87 \pm 0.80 \mu \mathrm{m}^{2}$ & $347.32 \pm 0.75 \mu \mathrm{m}^{2}$ \\
\hline $\begin{array}{r}\text { Number of Sides: } \\
\text { Hexagonal Cell Regularity } \\
\text { (Mean } \pm \text { SEM): }\end{array}$ & $61.95 \pm 0.04 \%$ & $5-9$ \\
\hline
\end{tabular}

Table 1. Comparative analysis of cells adjacent to the CJB.

The relationship of the SI to the hexagonal cell regularity, number of cell sides, and cell area was plotted in Graphs 15-17. The SI of the hexagonal cells in Graph 15 was sorted according to hexagonal cell regularity. The SD was again plotted to show the spread of cell SI values in relation to the CJB. These graphs were also plotted using a limited $y$-axis to illustrate more subtle trends. 


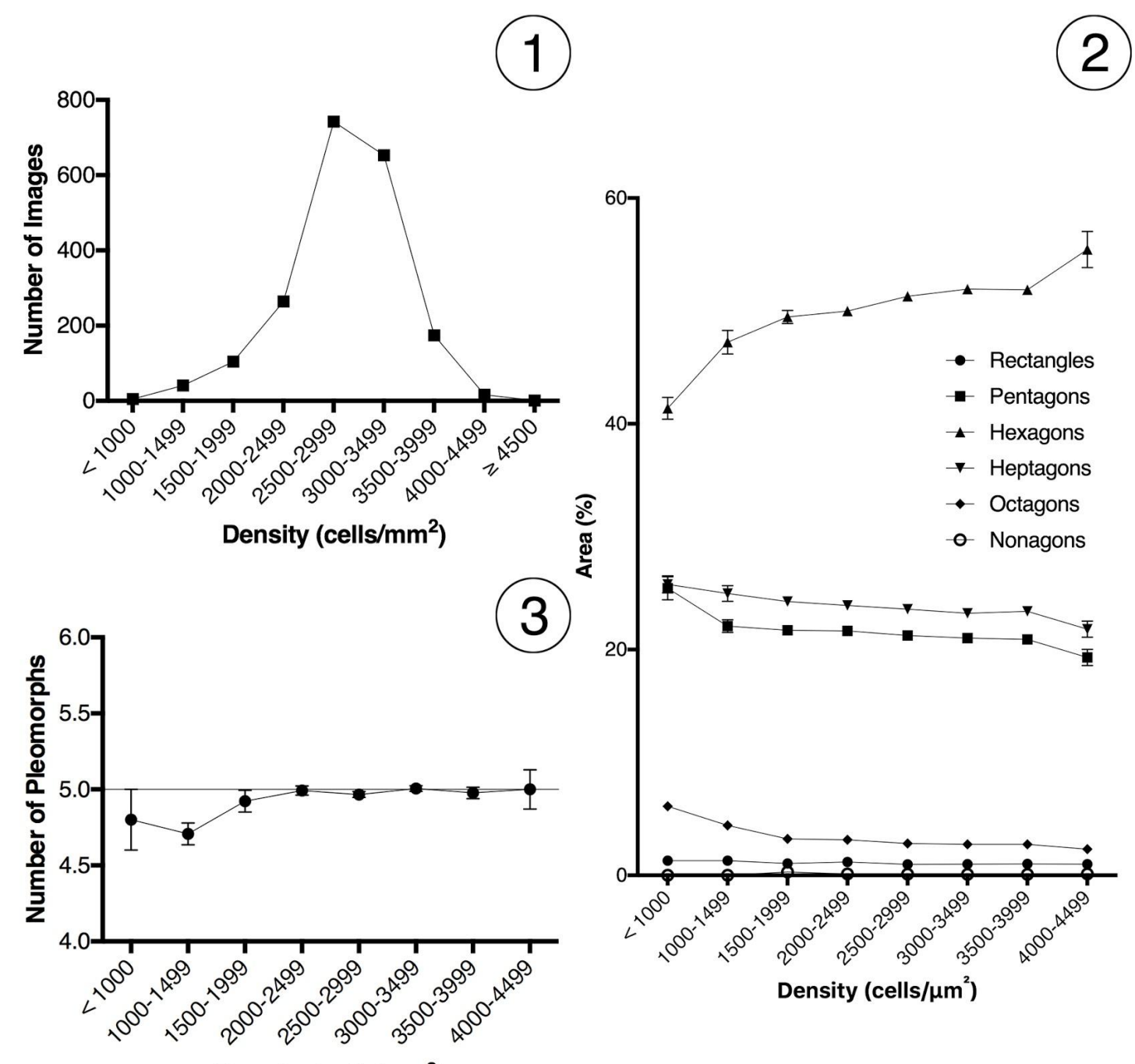

Density (cells $\left./ \mathrm{mm}^{2}\right)$

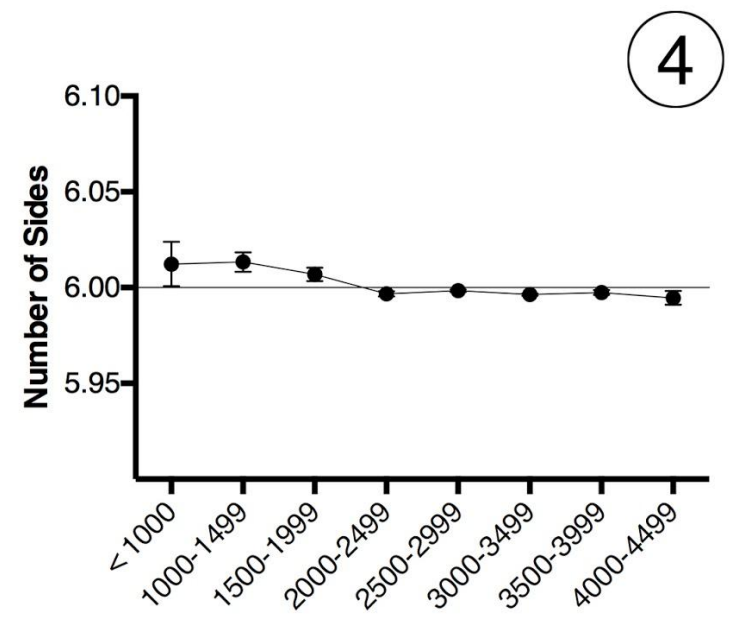

Density (cells $/ \mathrm{mm}^{2}$ )

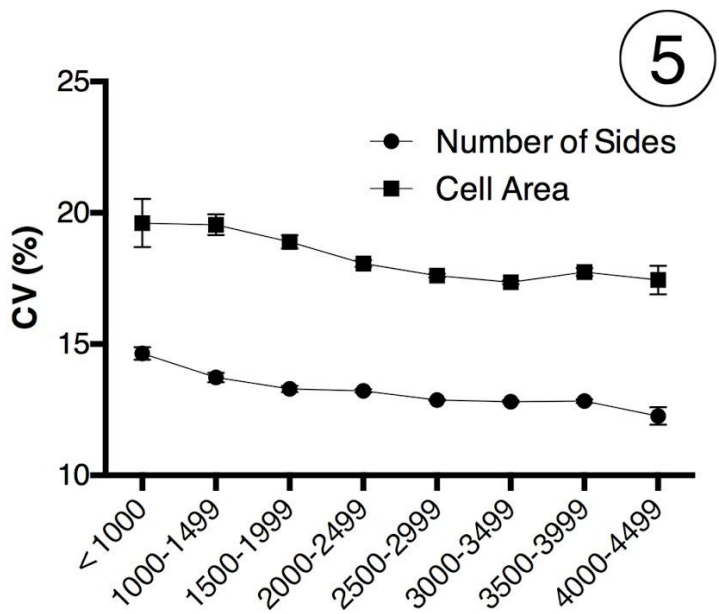

Density (cells $/ \mathrm{mm}^{2}$ )

Graphs 1-5. (1) Image ECD Distribution. (2) Image ECD vs. Image Pleomorph \% Area. (3) Image ECD vs. Image Number of Pleomorphs. (4) Image ECD vs. Image Number of Sides. (5) Image ECD vs. Image CV of the Number of Sides / Cell Area. Graphs 2-5: Mean \pm SEM. 

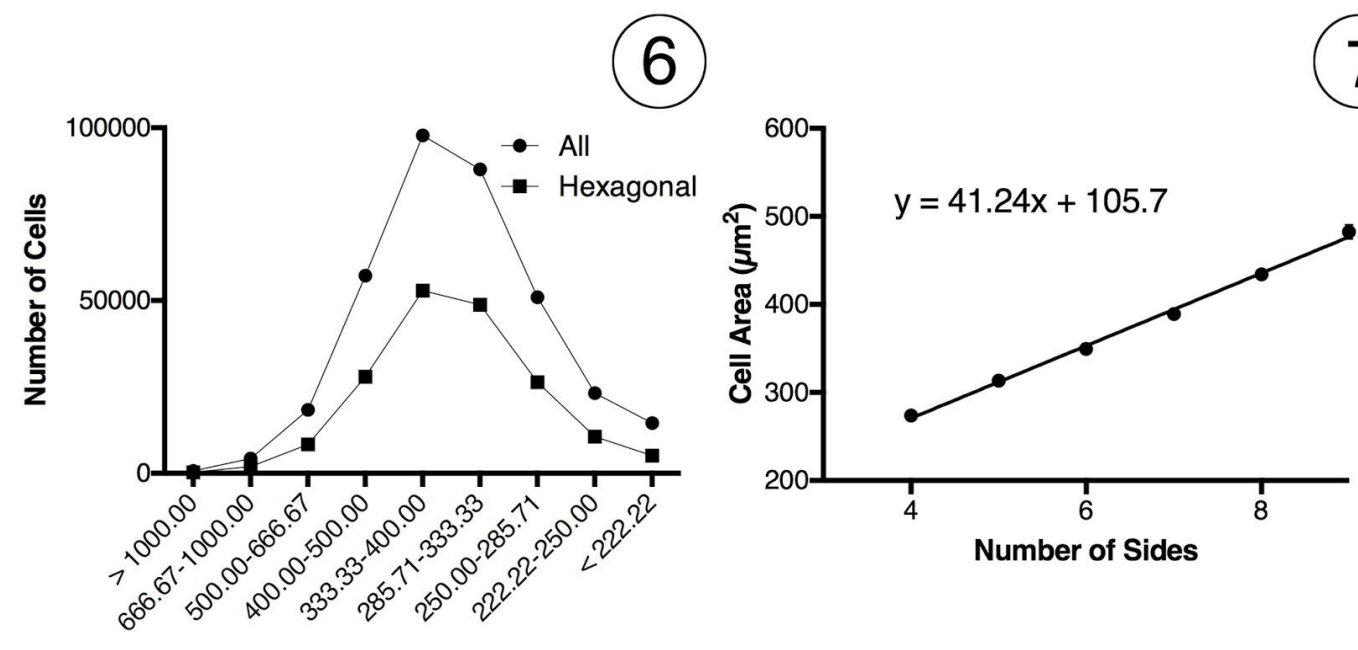

Cell Area $\left(\mu \mathrm{m}^{2}\right)$
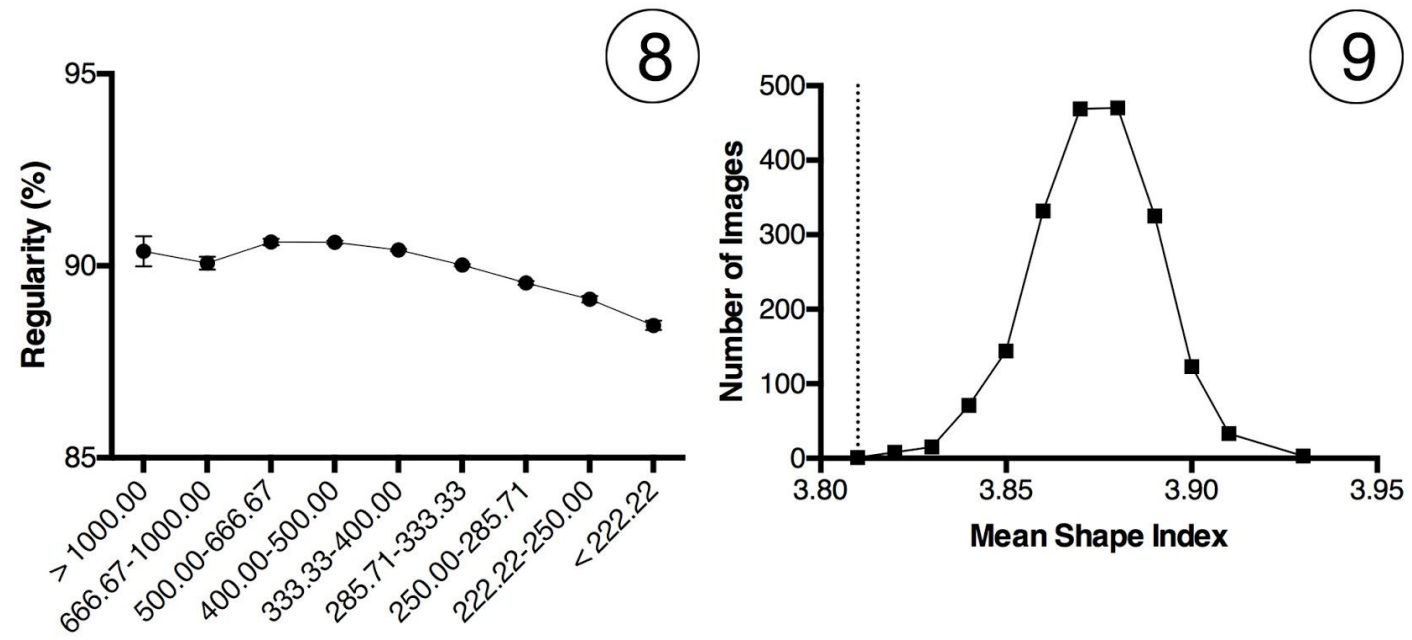

Hexagonal Cell Area $\left(\mu \mathrm{m}^{2}\right)$
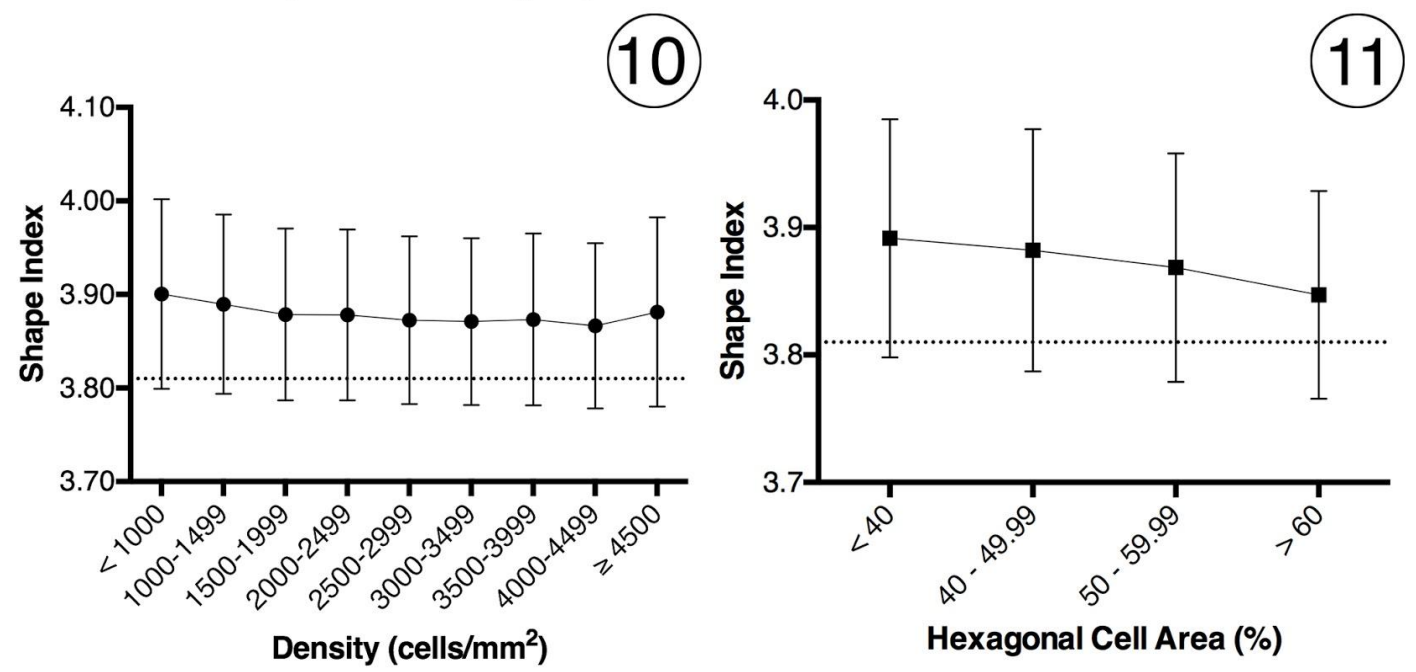

Graphs 6-11. (6) Cell Area Distribution. (7) Cell Area vs. Number of Cell Sides (with linear regression line). (8) Hexagonal Cell Area vs. \% Hexagonal Cell Regularity. (9) Image Shape Index Distribution. (10) Cell Shape Index vs. Image ECD. (11) Hexagonal Cell Shape Index vs. \% Image Hexagonal Cell Area. Graphs 7, 8: Mean \pm SEM. Graphs 10-11: Mean \pm SD. Graphs 9-11: dotted line $=$ CJB . 

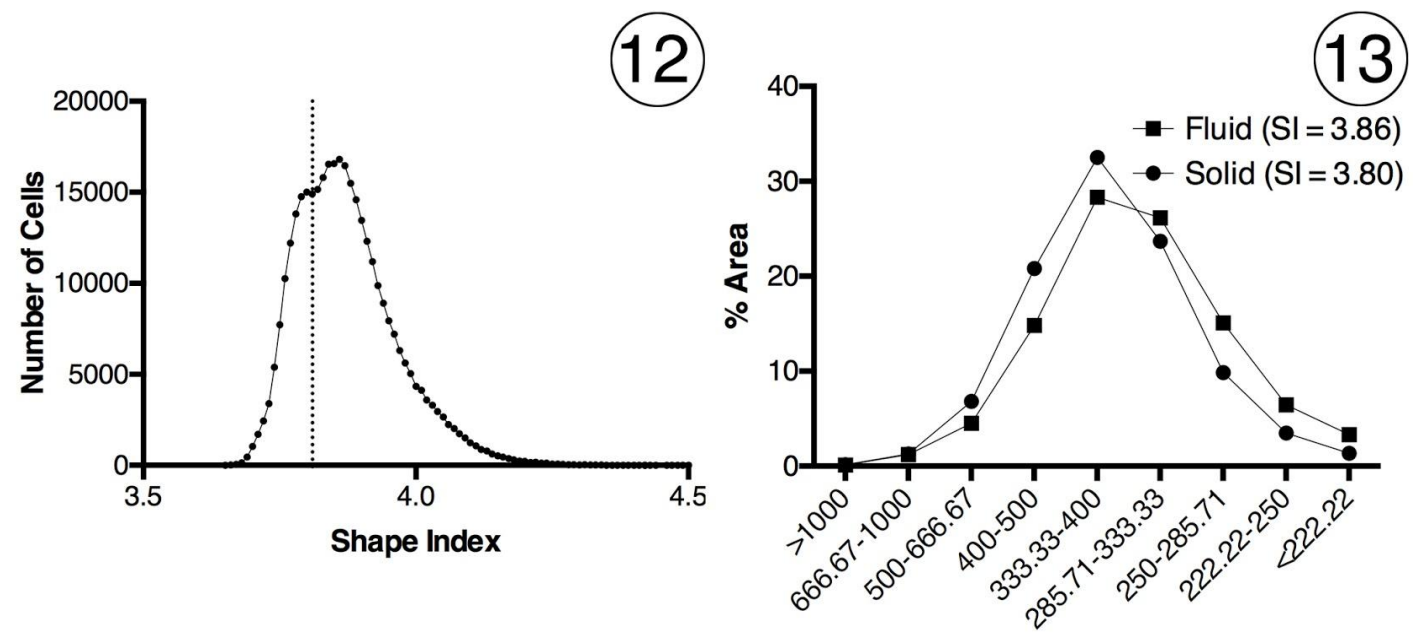

Cell Area $\left(\mu \mathrm{m}^{2}\right)$
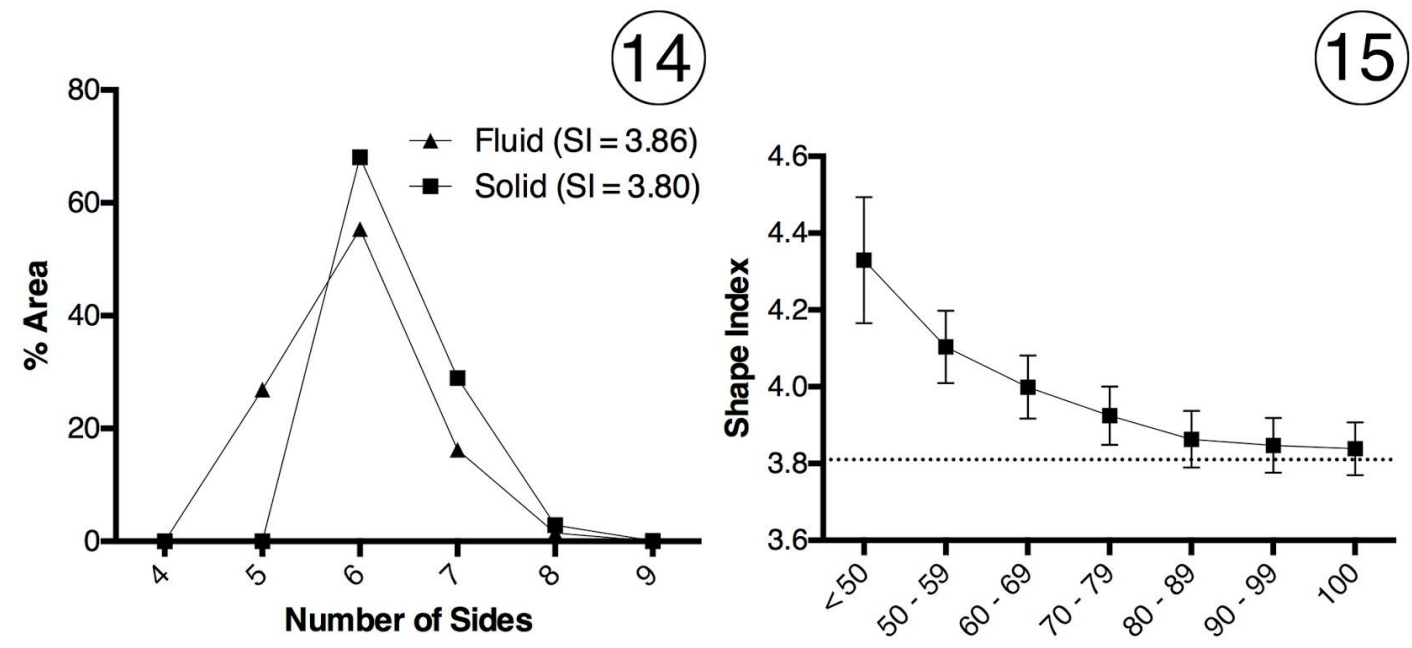

Hexagonal Cell Regularity (\%)
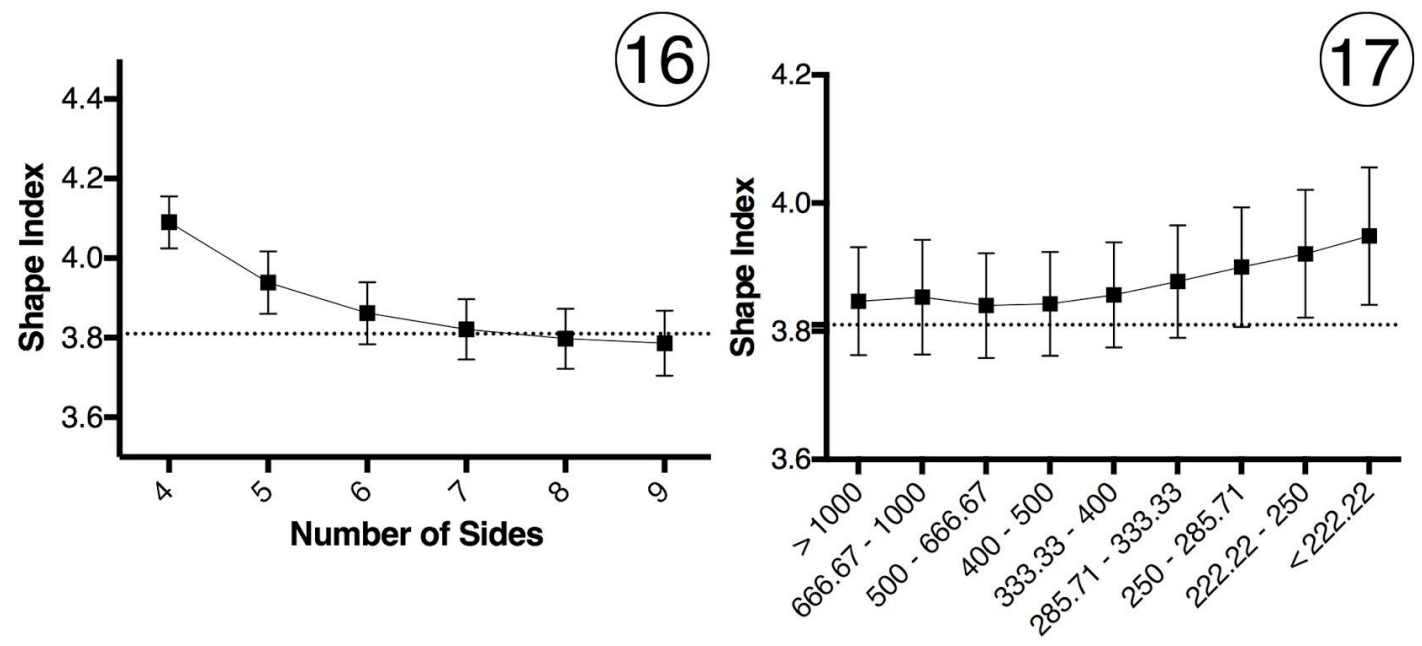

Cell Area $\left(\mu \mathrm{m}^{2}\right)$

Graphs 12-17. (12) Cell Shape Index Distribution. (13) Proportional areas of cells adjacent to the CJB. (14) Proportional cell areas of pleomorphs adjacent to the CJB. (15) Hexagonal Cell Regularity vs. Cell Shape Index. (16) Number of Cell Sides vs. Cell Shape Index. (17) Cell Area vs. Cell Shape Index. Graphs 15-17: Mean \pm SD. Graphs 12, 15-17: dotted line = CJB. 


\section{Discussion}

\subsection{Data Collection}

While the corneas used in this study were stored ex vivo in organ-culture, they were otherwise considered normal tissue that was almost all transplanted soon after imaging.

Swelling the intercellular spaces using hypotonic sucrose showed the convoluted basal and paracellular aspects of the cells in sharp relief using phase-contrast microscopy. The endothelial apical pole was also osmotically distorted, distinctly different from its in vivo appearance. These oedematous organ-cultured corneas had a folded Descemet's membrane so the endothelium shifted in and out of the focal plane, adding further complexity that made accurately segmenting images into measurable cells very challenging. There was nevertheless an observable mapping between the endothelial cell shapes and the computed VD, suggesting analogous spatial optimization processes.

\subsection{Endothelial Geometry and Topology}

In this study, the image ECD distribution was found to be not normal but skewed slightly toward higher densities (Graph 1). Likewise, cell areas for both all cells and the subset of hexagonal cells were skewed toward smaller cell areas (Graph 6).

As expected, hexagonal cells dominated the endothelial mosaics across the full ECD range. The proportional area contribution of hexagonal cells slowly increased with density (Graph 2), and simultaneously the areas of pentagonal, heptagonal and octagonal cells slowly decreased. There was a constant background population of rectangular cells comprising between $1-1.3 \%$ of the area, which also decreased in area as the density increased. The largely constant relative proportions of different polygons through the bulk of the ECD range shows self-similar topological coarsening [Cantat et al., 2013]. The increased proportional area of heptagons and octagons over rectangles and pentagons seen here may be partly due to the negative curvature of the posterior corneal surface [Weaire and Rivier, 1984], as well as the Lewis law.

Pleomorphic composition in proliferating tissues has been modelled to show that cell division processes alone will intrinsically converge on an irregular but predictable polygon distribution regardless of initial composition (28.9\% pentagons, $46.4 \%$ hexagons, $20.8 \%$ heptagons, and lesser numbers of other polygon types) [Gibson et al., 2006; Nagpal et al., 2008]. The corneal endothelium is the opposite of a proliferating tissue, where cells instead progressively die without replacement. While polygon proportions found in this study had some similarities to the model at low densities, there were quite different compositions throughout the bulk of the density range, suggesting that other factors must also contribute.

This notable stability in the number of hexagonal cells over the bulk of the range of ECD is predicted by both the Euler formula and the von Neumann-Mullins relation (Graph 4) [Cantat et al., 2013; Sánchez-Gutiérrez et al., 2016]. As found elsewhere [Lewis, 1926; Nagpal et al., 2008], even though the average number of cell sides was six there was still considerable diversity, with approximately 5 different cell shapes per image over the bulk of the ECD range (Graph 3). The average values plotted in Graph 4 additionally show that the mean topological charge was zero over the bulk of the ECD range in the corneal endothelium.

While the simplest measure of polymegathism is the mean and spread of the percentage areas of cells in the mosaic, this provides no information about the regularity of individual cells [Doughty, 1992]. Another measure is the CV of the cell area, but due to the inherent ambiguity in interpreting this its use has been discouraged [Doughty, 1989; Doughty, 1990]. As discussed previously, the ex vivo cell area CV values found 
in this study were lower than those reported in many other mostly in vivo studies, probably reflecting that the VD 'cell' shapes are composed of straight lines rather than the tortuous curves generated by edge-based cell segmentation techniques [Brookes, 2017a]. However, cell area CV values in the range reported here have been seen in studies of foetal and children's corneas [Farhan et al., 2014; Ko et al., 2001; Müller et al., 2000].

This current study has applied the CV to cell shape as well as size in order to further understand the complex topology of the endothelial mosaic. Both the mean cell size and shape CV decreased as ECD increased (Graph 5). The CV of number of sides has not been studied previously and showed that smaller cells (higher ECD) have less variation in shape as well as size.

Doughty introduced analysis of the area-side relationship as a means of comparing different endothelia that removes ambiguities inherent in the $\mathrm{CV}$ of cell area measurement [Doughty, 1990] and found a linear relationship between the cell area and the number of sides for cells with 4 - 8 sides $[38,60]$. This linear relationship was confirmed here using a far larger dataset (Graph 7 ), which fits the data extremely well extending to at least 9-sided cells, conforming to the Lewis law [Chiu, 1995].

As discussed previously, this study defined hexagonal cell regularity in a novel way [Brookes, 2017a], and while it was notably stable around $90 \%$ regular over the bulk of the cell size range, there was a decreasing trend where small cells had the least regularity, from a plateau around $400-500 \mu \mathrm{m}^{2}$ (Graph 8). The hexagon has a unique geometry where the length of each side is equal to the centre-vertex radius, balancing both internal and surface forces, and making it the most optimal configuration both geometrically and thermodynamically [Lecuit and Lenne, 2007; Honda, 1983; Rao et al., 1982; Yee et al., 1985].

\subsection{Shape Index and Tissue Phase}

The most recent SI analyses have all been performed using either computer modelling or in vitro cell culture with a range of animal cell lines [Bi et al., 2016; Park et al., 2016], so this study may be the first analysis of SI and the CJB in ex vivo human tissue.

The distribution of mean SI values in the endothelial images peaked at a mean (and median) of 3.87 (Graph 9). All but one of the 2000 images had a mean SI greater than the CJB at a SI of 3.81, showing that the endothelia were predominantly fluid. This was confirmed in Graph 10 across the entire ECD range, though the mean SI was largely independent of ECD with a very small decreasing trend. The SD plotted in Graph 10 shows that the SI of the cells comprising these endothelia spanned both sides of the CJB across the entire ECD range. The SI was however dependent on the percentage area of hexagonal cells in the image, where the higher the percentage of hexagonal cells the closer they were located to the CJB (Graph 11). Again, these hexagonal cell regions were comprised of cells with a range of SI's. The relative proportions of those below the CJB increased along with the proportional hexagonal cell area.

At the cell level (Graph 12), the SI showed a bimodal distribution that was perfectly centred at the CJB. The largest proportion of cells had Sl's greater than this boundary, confirming that endothelia dominated by them would be in a fluid phase. A smaller cell peak at SI's less than the boundary indicates a smaller cell population comprising solid endothelia. The shoulder in the graph at the CJB suggests some form of phase switching via shape transformation across this energy barrier, mostly toward more fluid mosaics.

Analysis of the cell populations under the two SI data points at the peaks of the bimodal distribution either side of the CJB at 3.80 (solid) and 3.86 (fluid) revealed quite different characteristics, as summarized in 
Table 1 and Graphs 13-14. Cells in the solid region contained a greater proportion of larger cells with six or more sides. The hexagonal cells were also more regularly shaped. There were no cells in this region with less than six sides. The dynamic balance of these mixtures suggests that shape and size change of only a small proportion of cells could radically modify the phase properties of the cell collective, and that this diverse morphological composition may be integral for the maintenance of endothelial structure and function.

SI was also dependent on hexagonal cell regularity (Graph 15), where the most regular hexagonal cells were closest to the CJB. The mean SI skated asymptotically toward the CJB, confirming that most endothelia were in a fluid phase, where rearranging their mosaics requires little energy. Beyond a regularity of about $80 \%$ the spread of values shows a proportion of cells crossing over the CJB. Hexagonal cell properties are uniquely balanced in relation to the $\mathrm{CJB}$, where small individual morphometric changes will have large effects on collective cell phase. The bottom of the range found in the dataset corresponded to the SI of a perfectly regular hexagon (3.72), well into the solid side of the boundary.

The SI was also dependent on both the number of cell sides (Graph 16) and cell area (Graph 17). Only cells with less than 6 sides had SI's above the CJB. While the mean hexagonal cell SI was also above the CJB, a proportion of these cells had SI's below it. Mean heptagonal cell SI was located very close to the CJB (3.82), and cells with more than seven sides had mean SI's below the CJB. However, in all but the two ten-sided cells in the dataset there was still a proportion with SI's on the fluid side of the CJB. While the smallest cells had the largest Sl's, this trend was lost than when averaged out at the image level, where individual endothelia were comprised of cells with a range of SI's (Graph 10).

\subsection{Centroidal Voronoi Tessellation}

This study computed the VD from a point cloud of cell centroids so was a form of Centroidal Voronoi Tessellation (CVT) [Du et al., 1999]. An optimized CVT topologically relaxes toward a uniform spatial distribution of centroids [Du et al., 2006] and applying Gersho's conjecture to a CVT with a large number of centroids shows it tends asymptotically toward comprising of only regular hexagons [Du and Wang, 2005]. This aligns with the Euler formula, where endothelia will tend to be dominated by regular hexagonal cells. Graphs 11 and 15-16 show that this arrangement shifts the cell collective closer to a solid phase.

The proportional polymorph area can also be compared to an idealized curve of all probable size-shape relationships using a CVT pathway graph [Sánchez-Gutiérrez et al., 2016]. This hypothetical framework can be used to compare the organization of a wide range of two-dimensional tissue architectures and suggests that they cannot present infinite organizations but are constrained to certain combinations of polygon distributions. Comparison of the data from this study with plots published by Sánchez-Gutiérrez et al. indicates that the ex vivo corneal mosaics are not fully optimized CVT's (data not shown) [Sánchez-Gutiérrez et al., 2016]. Such deviations from these idealized plots imply physical cellular constraints modifying the balance of forces within the tissue due to genetic or disease factors, thereby limiting tissue organization.

\subsection{Conclusion}

The data presented here shows that collectively the corneal endothelial mosaic acts as a glassy fluid, governed by well-characterized physical laws. The tissue is a form of viscous foam, with a cytoskeleton and intercellular junctional complexes tightly anchored to Descemet's membrane. Individual cell death transiently and locally increases collective cell entropy, so the endothelium must expend energy in order to rearrange and maintain function. Independent of ECD, this organization progressively tends toward a CVT 
with minimum free energy, comprised of a greater proportion of more uniformly sized cells with six or more sides. Becoming simultaneously more ordered and solid, each cell requires more energy to move. This rigidity is an emergent property that efficiently locks cells in place and maintains endothelial function. In vivo, this homeostasis is opposed by other factors such as age, environment, genetics, and pathology.

\section{References}

Alanko HI. Microcomputer analysis for corneal endothelial cell morphology. Acta Ophthalmol. 1983;61: 22939. PMID: 6880636.

Amann J, Holley GP, Lee S-B, Edelhauser HF. Increased endothelial cell density in the paracentral and peripheral regions of the human cornea. Am J Ophthalmol. 2003;135: 584-590. PMID: 12719063.

Bi D, Lopez JH, Schwarz JM, Manning ML. Energy barriers and cell migration in densely packed tissues. Soft Matter. 2014;10: 1885-1890. doi: 10.1039/c3sm52893f PMID: 24652538.

Bi J, Lopez JH, Schwarz JM, Manning ML. A density-independent rigidity transition in biological tissues. Nature Physics 2015;11: 1074-1080. doi: 10.1038/nphys3471

Bi D, Yang X, Marchetti MC, Manning ML. Motility-driven glass and jamming transitions in biological tissues. Phys. Rev X 2016;6: 021011. doi: 10.1103/PhysRevX.6.021011 PMID: 28966874; Pubmed Central PMCID: PMC5619672.

Bock M, Tyagi AK, Krefta J-U, Alt W. Generalized Voronoi tessellation as a model of two-dimensional cell tissue dynamics. Bull Math Biol. 2010;72: 1696-1731. doi: 10.1007/s11538-009-9498-3 PMID: 20082148. Bourne WM. Cellular changes in transplanted human corneas. Cornea. 2001;20: 560-569. PMID: 11473153. Brookes NH. Morphometry of organ-cultured corneal endothelium using Voronoi segmentation. Cell Tiss Bank. 2017a; 18: 167-183. doi: 10.1007/s10561-017-9622-9 PMID: 28374155.

Brookes NH. Measurements of ex vivo human corneal endothelium using Voronoi segmentation; 2017b [cited 2017 Dec 14]. Dataset: figshare [Internet]. Available from:

https://figshare.com/articles/Measurements_of_ex_vivo_human_corneal_endothelium_using_Voronoi_segm entation_/5701087

Cantat I, Cohen-Addad S, Elias F, Graner F, Höhler R, Pitois O, Rouyer F, Saint-Jalmes A. Foams: structure and dynamics. OUP Oxford; 2013; p31-32.

Charras GT, Coughlin M, Mitchison TJ, Mahadevan L. Life and times of a cellular bleb. Biophys J. 2008;94: 1836-1853. doi: 10.1529/biophysj.107.113605 PMID: 17921219; Pubmed Central PMCID: PMC2242777.

Chiu SN. Aboav-Weaire's and Lewis' laws - a review. Material Charact. 1995;34: 149-165.

Cunningham WJ, Moffatt SL, Brookes NH, Twohill HC, Pendergrast DGC, Stewart JM, et al. The New Zealand National Eye Bank Study: trends in the acquisition and storage of corneal tissue over the decade 2000-2009. Cornea. 2012;31: 538-45. doi: 10.1097/ICO.0b013e318222c3f2 PMID: 22314820.

da Fontoura Costa L. Characterizing polygonality in biological structures. Phys Rev E. 2006;73: 011913. doi: 10.1103/PhysRevE.73.011913 PMID: 16486191.

Dilts DM, Doughty MJ. A program for analyzing the morphological organization of tessellated cellular mosaics. Comput Biol Med. 1996;26: 409-418. PMID: 8889338. 
Doughty MJ. Toward a quantitative analysis of corneal endothelial cell morphology: a review of techniques and their application. Optom Vis Sci. 1989;66: 626-642. PMID: 2677883.

Doughty MJ. The ambiguous coefficient of variation: polymegethism of the corneal endothelium and central corneal thickness. Int Contact Lens Clin. 1990;17: 240-248. doi: 10.1016/0892-8967(90)90064-M

Doughty MJ. Concerning the symmetry of the 'hexagonal' cells of the corneal endothelium. Exp Eye Res. 1992;55: 145-154. PMID: 1397122.

Doughty MJ. Prevalence of 'non-hexagonal' cells in the corneal endothelium of young Caucasian adults, and their inter-relationships. Ophthalm Physiol Opt. 1998a;18: 415-422. PMID: 10023474.

Doughty MJ. Are there geometric determinants of cell area in rabbit and human corneal endothelial cell monolayers? Tiss Cell. 1998b;30: 537-544. PMID: 9839477.

Doughty MJ. Evaluation of possible error sources in corneal endothelial morphometry with a semiautomated noncontact specular microscope. Cornea. 2013;32: 1196-1203. doi: 10.1097/IC0.0b013e318295e72a PMID: 23792438.

Doughty MJ, Dilts DM. Identification of topographical variations in the sizes of cells in a monolayer application to corneal endothelium. Tiss Cell. 1994;26: 621-636. PMID: 8091424.

Drencken W, Hutzler S. Structure and energy of liquid foams. Adv Colloid Interface Sci. 2015;224: 1-16. doi: 10.1016/j.cis.2015.05.004 PMID: 26233494.

Du Q, Faber V, Gunzburger M. Centroidal Voronoi tessellations: applications and algorithms. SIAM Rev. 1999;41: 637-676. doi: 10.1137/S0036144599352836

Du Q, Emelianenko M, Ju L. Convergence of the Lloyd algorithm for computing centroidal Voronoi tessellations. SIAM J Num Anal. 2006;44: 102-119. doi: 10.1137/040617364

Du Q, Wang D. The optimal centroidal tessellations and the Gersho's conjecture in the three-dimensional space. Comp Math Appl. 2005;49: 1355-1373. doi: 10.1016/j.camwa.2004.12.008

Elbaz U, Mireskandari K, Tehrani N, Shen C, Khan MS, Williams S, et al. Corneal endothelial cell density in children: normative data from birth to 5 years old. Am J Ophthalmol. 2017;173: 134-138. doi:

10.1016/j.ajo.2016.09.036 PMID: 27746297.

Farhan HA, Albaow W, Masoud W. Normal corneal endothelial morphology of healthy Saudi children aged 712 years. J Egypt Ophthalmol Soc. 2014;107: 63-66.

Geroski DH, Matsuda M, Yee RW, Edelhauser HF. Pump function of the human corneal endothelium: effects of age and corneal guttata. Ophthalmol. 1985;92: 759-763. PMID: 2412197.

Gibson MC, Patel AB, Nagpal R, Perrimon N. The emergence of geometric order in proliferating metazoan epithelia. Nature. 2006;442: 1038-1041. doi: 10.1038/nature05014 PMID: 16900102.

Haeger A, Krause M, Wolf K, Friedl P. Cell jamming: Collective invasion of mesenchymal tumor cells imposed by tissue confinement. Biochimica et Biophysica Acta 2014;1840: 2386-2395. doi:

10.1016/j.bbagen.2014.03.020 PMID: 24721714.

Harrison TA, He Z, Boggs K, Thuret G, Liu H-X, Defoe DM. Corneal endothelial cells possess an elaborate multipolar shape to maximize the basolateral to apical membrane area. Mol Vis. 2016;22: 31-39. PMID: 27081293; Pubmed Central PMCID: PMC4814271.

He Z, Forest F, Gain P, Rageade D, Bernard A, Acquart S, et al. 3D map of the human corneal endothelial cell. 
Sci Rep. 2016;6: 29047. doi: 10.1038/srep29047 PMID: 27381832; Pubmed Central PMCID: PMC4933894. Honda H. Geometrical models for cells in tissues. Int Rev Cytol. 1983;85: 191-248. PMID: 6347934. Honda $\mathrm{H}$, Nagai T. Cell models lead to understanding of multi-cellular morphogenesis consisting of successive self-construction of cells. J Biochem. 2015; 157: 129-136. doi: 10.1093/jb/mvu088 PMID: 25552548.

Honda H, Ogita Y, Higuchi S, Kani K. Cell movements in a living mammalian tissue: Long-term observation of individual cells in wounded corneal endothelia of cat. J Morphol. 1982; 174: 25-39. doi:

10.1002/jmor.1051740104 PMID: 7143447.

Joyce NC. Cell cycle status in human corneal endothelium. Exp Eye Res. 2005;81: 629-638. doi: 10.1016/j.exer.2005.06.012 PMID: 16054624.

Joyce NC. Proliferative capacity of corneal endothelial cells. Exp Eye Res. 2012;95: 16-23. doi: 10.1016/j.exer.2011.08.014 PMID: 21906590; Pubmed Central PMCID: PMC3261346.

Kirk AH, Hassard DTR. Supravital staining of the corneal endothelium and evidence for membrane on its surface. Can J Ophthalmol. 1969;4: 405-415. PMID: 4186214.

Kim JH, Serra-Picamal X, Tambe DT, Zhou EH, Park CY, Sadati M, et al. Propulsion and navigation within the advancing monolayer sheet. Nat. Mat. 2013;12: 856-863. doi: 10.1038/nmat3689 PMID: 23793160; Pubmed Central PMCID: PMC3750079.

Ko M-K, Park WK, Lee JH, Chi JG. A histomorphometric study of corneal endothelial cells in normal human fetuses. Exp Eye Res. 2001;72: 403-409. doi: 10.1006/exer.2000.0964 PMID: 11273668.

Lecuit T, Lenne P-F. Cell surface mechanics and the control of cell shape, tissue patterns and morphogenesis. Nature Rev Mol Cell Biol. 2007;8: 633-644. doi: 10.1038/nrm2222 PMID: 17643125.

Lewis FT. The effect of cell division on the shape and size of hexagonal cells. Anat Rec. 1926;33: 331-335.

MacPherson RD, Srolovitz DJ. The von Neumann relation generalized to coarsening of three-dimensional microstructures. Nature. 2007;446: 1053-1055. doi: 10.1038/nature05745

Mason JK, Lazar EA, MacPherson RD, Srolovitz DJ. Statistical topology of cellular networks in two and three dimensions. Phys Rev E. 2012;86: 051128. doi: 10.1103/PhysRevE.86.051128 PMID: 23214759.

Mishima S. Clinical investigations on the corneal endothelium. Am J Ophthalmol 1982;93: 1-29. PMID: 6801985.

Müller A, Doughty MJ, Wright L. Reassessment of the corneal endothelial cell organisation in children. $\mathrm{Br} \mathrm{J}$ Ophthalmol. 2000;84: 692-696. PMID: 10873975; Pubmed Central PMCID: PMC1723537.

Müller A, Doughty MJ. Assessments of corneal endothelial cell density in growing children and its relationship to horizontal corneal diameter. Optom Vis Sci. 2002;79: 762-770. PMID: 12512684.

Murphy C, Alvarado J, Jusrer R, Maglio M. Prenatal and postnatal cellularity of the human corneal endothelium. A quantitative histologic study. Invest Ophthalmol Vis Sci. 1984;25: 312-322. PMID: 6698749.

Nagpal R, Patel A, Gibson MC. Epithelial topology. BioEssays 2008;30: 260-266. doi: 10.1002/bies.20722 PMID: 18293365.

Nishimura JK, Hodge DO, Bourne WM. Initial endothelial cell density and chronic endothelial cell loss rate in corneal transplants with Late Endothelial Failure. Ophthalmology 1999;106: 1962-1965. doi: 


\subsection{6/S0161-6420(99)90409-8 PMID: 10519593.}

Norman LL, Brugés J, Sengupta K, Sens P, Aranda-Espinoza H. Cell blebbing and membrane area homeostasis in spreading and retracting cells. Biophys J. 2010;99: 1726-1733. doi:

10.1016/j.bpj.2010.07.031 PMID: 20858416; Pubmed Central PMCID: PMC2944031.

Norman L, Sengupta K, Aranda-Espinoza H. Blebbing dynamics during endothelial cell spreading. Eur J Cell Biol. 2011;90: 37-48. doi: 10.1016/j.ejcb.2010.09.013 PMID: 21087809.

Park J-A, Atia L, Mitchel JA, Fredburg JJ, Butler JP. Collective migration and cell jamming in asthma, cancer and development. J Cell Sci. 2016;129: 3375-3383. doi: 10.1242/jcs.187922 PMID: 27550520; Pubmed Central PMCID: PMC5047682.

Park A-H, Kim JH, Bi D, Mitchel JA, Qazvini NT, Tantisira K, et al. Unjamming and cell shape in the asthmatic airway epithelium. Nature Materials 2015;14: 1040-1049. doi: 10.1038/nmat4357 PMID: 26237129; Pubmed Central PMCID: PMC4666305.

Pegoraro AF, Fredberg JJ, Park J-A. Problems in biology with many scales of length: Cell-cell adhesion and cell jamming in collective cellular migration. Exp. Cell Res. 2016;343: 54-59. doi:

10.1016/j.yexcr.2015.10.036 PMID: 26546401; Pubmed Central PMCID: PMC4851584.

Pels L. Organ culture: the method of choice for preservation of human donor corneas. $\mathrm{Br} \mathrm{J}$ Ophthalmol. 1997; 81: 523-525. PMID: 9290360; Pubmed Central PMCID: PMC1722261.

Plateau J. Statique expérimentale et théorique des liquides soumis aux seules forces moléculaires. Paris: Gauthier-Villars; 1873.

Price MO, Price FW. Endothelial cell loss after Descemet Stripping with Endothelial Keratoplasty. Influencing factors and 2-year trend. Ophthalmol. 2008;115: 857-865. doi: 10.1016/j.ophtha.2007.06.033 PMID: 17868873.

Rao GN. Morphometry of corneal endothelium. Cornea. 1985;3: 153-154. PMID: 6544191.

Rao GN, Lohman LE, Aquavella JV. Cell size-shape relationships in corneal endothelium. Invest Ophthalmol Vis Sci. 1982;22: 271-274. PMID: 7035396.

Reinhart-King CA, Dembo M, Hammer DA. The dynamics and mechanics of endothelial cell spreading. Biophys J. 2005;89: 676-689. doi: 10.1529/biophysj.104.054320 PMID: 15849250; Pubmed Central PMCID: PMC1366566.

Ruusuvaara P, Vesti E, Koskela E, Setälä K. One hundred-year-old human corneal and transplanted corneal graft endothelium. Acta Ophthalmol. 2015;93: 575-578. doi: 10.1111/aos.12715 PMID: 25833150.

Sadati M, Qazvini NT, Krishnan R, Park CY, Fredberg JJ. Collective migration and cell jamming. Differentiation. 2013;86: 121-125. doi: 10.1016/j.diff.2013.02.005 PMID: 23791490; Pubmed Central PMCID: PMC3795803.

Sánchez-Gutiérrez D, Tozluoglu M, Barry JD, Pascual A, Mao Y, Escudero LM. Fundamental physical cellular constraints drive self-organization of tissues. EMBO J. 2016;35: 77-88. doi: 10.15252/embj.201592374 PMID: 26598531; Pubmed Central PMCID: PMC4718000.

Schimmelpfennig BH. Direct and indirect determination of nonuniform cell density distribution in human corneal endothelium. Invest Ophthalmol Vis Sci. 1984;25: 223-229. PMID: 6365828.

Schultz RO, Matsuda M, Yee RW, Edelhauser HF, Schultz KJ. Corneal endothelial changes in Type I And Type 
bioRxiv preprint doi: https://doi.org/10.1101/236406; this version posted February 28, 2018. The copyright holder for this preprint (which was not certified by peer review) is the author/funder. All rights reserved. No reuse allowed without permission.

II Diabetes Mellitus. Am J Ophthalmol 1984;98: 401-410. PMID: 6486211.

Sperling S. Endothelial cell density in donor corneas. Acta Ophthalmol. 1980;58: 278-282. PMID: 6994426.

Trepat X, Fredberg JJ. Plithotaxis and emergent dynamics in collective cellular migration. Trends Cell Biol. 2011;21: 638-646. doi: 10.1016/j.tcb.2011.06.006 PMID: 21784638; Pubmed Central PMCID: PMC3202659.

Vitorino P, Hammer M, Kim J, Meyer T. A steering model of endothelial sheet migration recapitulates monolayer integrity and directed collective migration. Mol Cell Biol. 2011;31: 342-350. doi:

10.1128/MCB.00800-10 PMID: 20974808; Pubmed Central PMCID: PMC3019974.

Weaire D, Rivier N. Soap, cells and statistics-random patterns in two dimensions. Contemp Phys. 1984;25: 59-99. doi: $10.1080 / 00107518408210979$

Wilson SE, Bourne WM. Corneal preservation. Surv Ophthalmol. 1989;33: 237-259. PMID: 2652359.

Wilson RS, Roper-Hall MJ. Effect of age on the endothelial cell count in the normal eye. $\mathrm{Br} \mathrm{J}$ Ophthalmol. 1982;66: 513-515. PMID: 7104267; Pubmed Central PMCID: PMC1039838.

Wörner $\mathrm{CH}$, Olguín A, Ruíz-Garcían JL, Garzón-Jiménez N. Cell pattern in adult human corneal endothelium. PLoS One. 2011;6: e19483. doi: 10.1371/journal.pone.0019483 PMID: 21602935; Pubmed Central PMCID: PMC3094353.

Yee RW, Matsuda M, Schultz RO, Edelhauser HF. Changes in the normal corneal endothelial cellular pattern as a function of age. Curr Eye Res. 1985;4: 671-678. PMID: 4028790. 